\title{
SARS-CoV-2 Vaccine and Thrombosis: An Expert Consensus on Vaccine-Induced Immune Thrombotic Thrombocytopenia
}

\author{
Ismail Elalamy ${ }^{1,2,3}$ Grigoris Gerotziafas ${ }^{1,2}$ Sonia Alamowitch ${ }^{4,5}$ Jean-Pierre Laroche ${ }^{6,7}$ \\ Patrick Van Dreden ${ }^{2}$ Walter Ageno ${ }^{8}$ Jan Beyer-Westendorf ${ }^{9}$ Alexander T. Cohen ${ }^{10}$ David jimenez ${ }^{11}$ \\ Benjamin Brenner ${ }^{3,12}$ Saskia Middeldorp ${ }^{13}$ Patrice Cacoub ${ }^{14,15,16,17}$ Scientific Reviewer Committee*
}

${ }^{1}$ Hematology and Thrombosis Center, Hôpital Tenon, Hôpitaux Universitaires de l'Est Parisien, Assistance Publique Hôpitaux de Paris, Faculté de Médecine, Sorbonne Université, Paris, France

2 Research Group "Cancer, Haemostasis and Angiogenesis," INSERM U938, Centre de Recherche Saint-Antoine, Institut Universitaire de Cancérologie, Faculty of Medicine, Sorbonne University, Paris, France

${ }^{3}$ Department of Obstetrics and Gynaecology, The First I.M. Sechenov Moscow State Medical University, Moscow, Russia

${ }^{4}$ AP-HP, Service des Urgences cérébro-vasculaires, Hôpital PitiéSalpétrière, Paris, France

${ }^{5}$ Centre de recherche de Saint Antoine, INSERM, UMRS 938, Sorbonne Université Paris, France

${ }^{6}$ Service de médecine vasculaire, hôpital Saint-Éloi, CHU de Montpellier, Montpellier cedex 5, France

${ }^{7}$ Médipôle, 1139, chemin du Lavarin, Avignon, France

${ }^{8}$ Department of Emergency Medicine and Thrombosis Center, Ospedale di Circolo and Department of Medicine and Surgery, University of Insubria, Varese, Italy

${ }^{9}$ Department of Medicine I, Division of Haematology and Haemostaseology, University Hospital Carl Gustav Carus, Technische Universität Dresden, Dresden, Germany

10 Department of Haematological Medicine, Guys and St Thomas' NHS Foundation Trust, King's College London, London, United Kingdom
Address for correspondence Ismail Elalamy, MD, PhD, Hematology and Thrombosis Center, Tenon University Hospital, Département Médico-Universitaire de Biologie et Génomique Médicales (BioGeM), Medecine Sorbonne University, INSERM UMR S-938, Hôpitaux Universitaires de l'Est Parisien (HUEP) APHP.6, 4, rue de la Chine 75970 Paris Cedex 20, France (e-mail: ismail.elalamy@aphp.fr).

\footnotetext{
11 Respiratory Department, Ramon y Cajal Hospital (IRYCIS), CIBER Enfermedades Respiratorias (CIBERES), Madrid, Spain

12 Department of Hematology, Rambam Health Care Campus, Haifa, Israel

13 Department of Internal Medicine, Radboud Institute for Health Sciences, Radboud University Medical Centre, Nijmegen, The Netherlands

${ }^{14}$ Department of Internal Medicine and Clinical Immunology, AP-HP, Groupe Hospitalier Pitié-Salpêtrière, Centre de Référence des Maladies Auto-Immunes Systémiques Rares, Centre de Référence des Maladies Auto-Inflammatoires et de l'Amylose inflammatoire, Paris, France

${ }^{15}$ Institut National de la Santé et de la Recherche Médicale, INSERM, Paris, France

${ }^{16}$ CNRS, FRE3632, Paris, France

${ }^{17}$ Inflammation-Immunopathology-Biotherapy Department (DHU i2B), Sorbonne Université, UPMC Univ Paris 06, Paris, France
}

Thromb Haemost 2021;121:982-991.

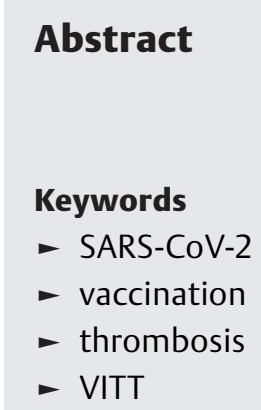

Historically, the vaccination strategies developed in the second half of the 20th century have facilitated the eradication of infectious diseases. From the onset of COVID-19 pandemic to the end of April 2021, more than 150 million cases and 3 million deaths were documented worldwide with disruption of the economic and social activity, and with devastating material, physical, and psychological consequences. Reports of unusual and severe thrombotic events, including cerebral and splanchnic venous thrombosis and other autoimmune adverse reactions, such as immune thrombocytopenia or thrombotic microangiopathies in connection with some of the SARS-CoV-2 vaccines, have caused a great deal of concern within the

Scientific Reviewer Committee Members: Gregory Y. H. Lip, Michael Makris, Sam Schulman, Wolfgang Siess, Christian Weber

received

April 22, 2021

accepted after revision

May 3, 2021

published online

May 4, 2021
DOI https://doi.org/

10.1055/a-1499-0119. ISSN 0340-6245.

\author{
(c) 2021. The Author(s). \\ This is an open access article published by Thieme under the terms of the \\ Creative Commons Attribution-NonDerivative-NonCommercial-License, \\ permitting copying and reproduction so long as the original work is given \\ appropriate credit. Contents may not be used for commercial purposes, or \\ adapted, remixed, transformed or built upon. (https://creativecommons.org/ \\ licenses/by-nc-nd/4.0/) \\ Georg Thieme Verlag KG, Rüdigerstraße 14, 70469 Stuttgart, \\ Germany
}


population and the medical community. This report is intended to provide practical answers following an overview of our knowledge on these thrombotic events that are extremely rare but have serious consequences. Vaccine hesitancy threatens to reverse the progress made in controlling vaccine-preventable diseases. These adverse events must be put into perspective with an objective analysis of the facts and the issues of the vaccination strategy during this SARS-CoV-2 pandemic. Health care professionals remain the most pertinent advisors and influencers regarding vaccination decisions; they have to be supported to provide reliable and credible information on vaccines. We need to inform, reassure, and support our patients when the prescription is made. Facing these challenges and observations, a panel of experts express their insights and propose a tracking algorithm for vaccinated patients based on a 10point guideline for decision-making on what to do and not to do.

\section{Introduction}

After SARS-CoV-2 vaccination campaign initiation, European reports of rare, unusual, and severe thrombotic events, such as cerebral venous sinus thrombosis (CVST) and splanchnic venous thrombosis (SVT), and other autoimmune adverse reactions such as immune thrombocytopenia or thrombotic microangiopathies in connection with some of the SARS-CoV-2 vaccines, have caused a lot of worry and even a panic turmoil within the population and the medical community. These events must be put into perspective with an objective analysis of the facts and the issues of the vaccination strategy during this pandemic. Among its many facets, coronavirus disease 2019 (COVID-19) is associated with an increased risk of vascular disease with often life-threatening thrombotic manifestations. After an overview of our knowledge on these extremely rare but extremely serious thrombotic events, this manuscript intends to provide practical answers and attitudes. Of course, many points still need to be clarified through cooperative, multinational, prospective studies. In the meantime, we must learn to better cope with the consequences of these rare, albeit worrisome complications through continuous pharmacovigilance and appropriate management.

\section{Different Types of SARS-CoV-2 Vaccines: Facts and Implications}

Historically, the vaccination strategies developed in the second half of the 20th century have facilitated the eradication of infectious diseases such as poliomyelitis, diphtheria, and smallpox, and have considerably reduced the incidence of childhood illnesses, notably measles, mumps, and rubella. The battle, however, has never been completely won. For example, there has been a $30 \%$ increase in measles cases worldwide, and in England the number of cases of measles and mumps has doubled in recent years. The reasons that people choose to not be vaccinated are complex. The World Health Organization (WHO) recently listed vaccine hesitancy as one of the 10 greatest threats to global health. ${ }^{1}$ It identified the main reasons for hesitancy as the difficulties of access to vaccines and the lack of confidence. ${ }^{2}$ Health care professionals remain the most pertinent advisors and influencers regarding vaccination decisions; they need to be supported to provide reliable and credible information on vaccines. Vaccine hesitancy-the reluctance or refusal to be vaccinated despite the availability of vaccines-threatens to reverse the progress made in controlling vaccine-preventable diseases. Vaccination is one of the most cost-effective ways of preventing disease. It currently prevents 2 to 3 million deaths per year, and an additional 1.5 million could be avoided with improvement of global vaccination coverage. $^{2}$

From the onset of COVID-19 pandemic to April 2021, more than 150 million cases and 3 million deaths were documented worldwide with disruption of the economic and social activity, and with devastating material, physical, and psychological consequences. ${ }^{3,4}$ Unfortunately, a large proportion of the public is still hesitant to accept the dangers associated with SARS-CoV-2, comparing it with influenza epidemics from the past, ignoring the fact that the death toll continues to rise globally despite strict hygiene measures and lock-downs. The rapid availability of an effective vaccine for limiting viral transmission and serious forms of the disease has emerged as the only real solution for controlling this pandemic ${ }^{5}$ (-Fig. 1). The development of antibodies directed against one part of the spike protein (the protein that enables SARS-CoV-2 to bind to the membrane receptor for angiotensin-converting enzyme-2 and thus promote viral invasion) is the strategy chosen by most vaccine developers. It is necessary to keep in mind that these vaccines also promote development of cellular immunity via the action on dendritic cells and $\mathrm{T}$ cells such as cytotoxic $\mathrm{T}$ and T-helper lymphocytes. 6,7

To date, 240 vaccine candidates have been registered by WHO: 63 in the clinical evaluation phase, 177 in the preclinical phase, and 11 that are authorized in at least one country.

\section{Thrombosis and Vaccines: Very Rare Events}

An association between the AstraZeneca vaccine (Vaxzevria/ ChAdOx1 nCoV-19/AZD1222) and rare cases of thrombosis have been recently reported in United Kingdom (UK) and Europe. ${ }^{8-11}$ In contrast to adenoviral vaccines (AstraZeneca and Johnson \& Johnson/Janssen), no CVST and SVT cases have been linked to mRNA vaccines (Comirnaty/BioNTech/Pfizer and mRNA-1273/Moderna). ${ }^{10}$ In UK, Germany, Austria, and Norway, thrombosis in unusual locations, such as cerebral 


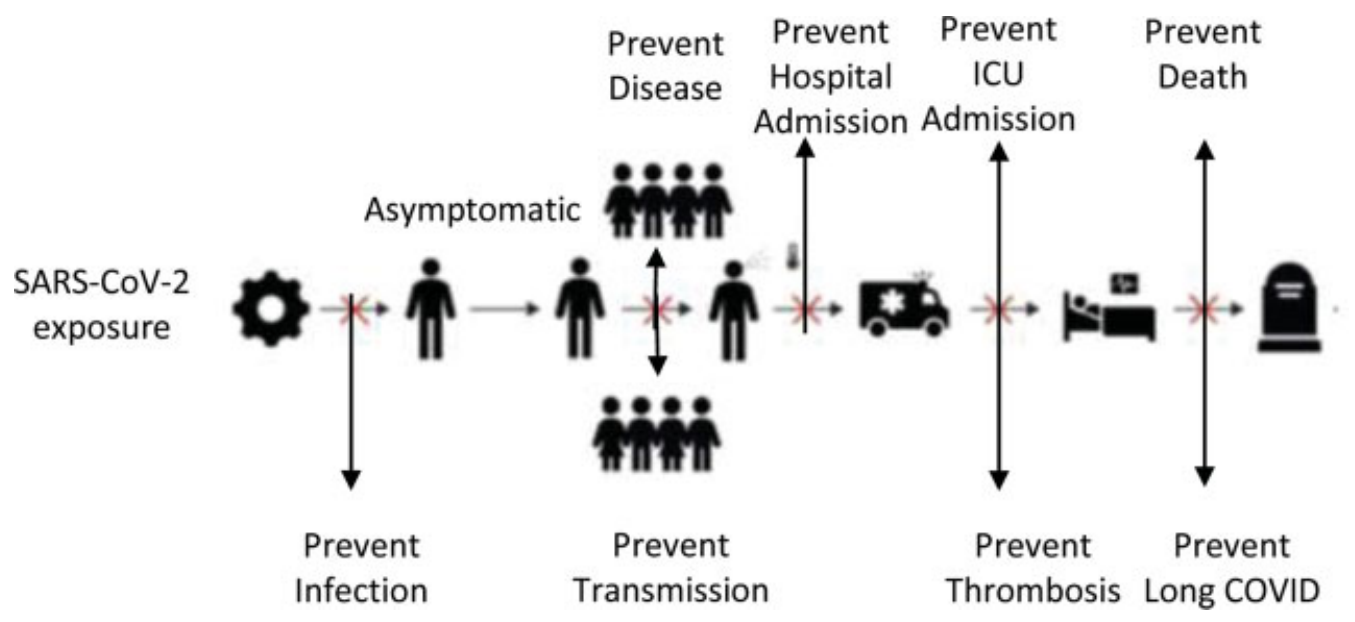

Fig. 1 SARS-CoV-2 vaccination objectives. ${ }^{5}$ Among multiple objectives and benefits of SARS-CoV-2 vaccination, significant prevention of thrombosis must also be taken into account.

venous sinus, splanchnic vein or pulmonary thrombosis, have been reported in the days following vaccination (within 4-24 days). ${ }^{12-14}$ The authors have proposed grouping them under the acronym "VITT" (vaccine-induced immune thrombotic thrombocytopenia). Indeed, these episodes of venous thrombosis were associated with low platelet levels and a strong increase in D-dimers with normal or low fibrinogen levels. Of the 11 reported cases in Germany and Austria, 10 were CVST that occurred in young women (9/11, aged 22-49 years). ${ }^{13}$ These CVST cases were associated with other forms of thrombosis, including pulmonary emboli (PE; 3 cases), SVT ( 3 cases), and other unusual thromboses ( 4 cases). Six of the patients died. Two of the women had autoimmune disease. ${ }^{13}$ The Norwegian authors reported the occurrence of five cases of CVST with severe thrombocytopenia in health care professionals ${ }^{14}$; three patients died. They were mainly young women $(4 / 5$, aged $32-54$ years $) .{ }^{14}$ These thrombotic episodes occurred 7 to 10 days after injection of the AstraZeneca vaccine. At that time, close to 133,000 people had received a single dose of this vaccine in Norway. ${ }^{14}$ In the largest cohort reported in UK with 23 cases, they were 14 women (22-71 years old) and 9 men (21-77 years old). ${ }^{12} \mathrm{~A}$ total of 13 thrombotic episodes were related to CVST, 6 cases to PE, and 4 cases to SVT. ${ }^{12}$ Seven patients died. It is not known whether these patients had other risk factors of thrombosis (e.g., use of birth control pills, comorbidity, acquired or inherited thrombophilia, overweight, or obesity).

On April 4, 2021, with more than 40 million vaccinated individuals, 169 cases of CVST and 53 cases of SVT were reported on the European EudraVigilance database. ${ }^{15-17}$ On April 17, 2021, data from the UK pharmacovigilance, the Medicines and Healthcare products Regulatory Agency (MHRA) Yellow Card Scheme, reported 168 severe thrombotic events (including 77 CVST) among 22 million people who received the first dose of AstraZeneca vaccine. ${ }^{8}$ The overall incidence is around 8 per 1 million doses. ${ }^{8}$ These events occurred in 93 women and 75 men aged 18 to 93 years and the overall case fatality rate was $19 \%$ with 32 deaths. ${ }^{8}$ On April 23, 2021, the Vaccine Adverse Event Reporting System
(VAERS) database in the United States recorded 161 classical thrombotic events during COVID-19 vaccination campaign with 5 CVST without thrombocytopenia among 125 million Moderna vaccinated individuals and 154 million doses of BioNTech/Pfizer vaccine administered. ${ }^{18}$ In the last update, on April 15, 2021, the French Agency reported a total of 27 cases of severe thrombosis (24 CVST, 2 SVT, and 1 PE with disseminated intravascular coagulopathy) in around $3,300,000$ people who received a first injection of the AstraZeneca vaccine. ${ }^{19}$ The sex ratio was around 1 (13 women/14 men) and the mean age was 63 years old. ${ }^{19}$ No such severe thrombotic case was reported among 12 million doses of BioNTech/Pfizer vaccine and 1.5 million doses of Moderna vaccine to date. ${ }^{19}$ The incidence of these severe venous thrombotic events therefore appears particularly low, $\sim 1 / 100,000 .{ }^{9}$ These types of incidents have not been reported in India thus far, despite a particularly high use of the AstraZeneca vaccine. Some cases of unrelated acute myocardial infarction episodes were reported but a review is being conducted by India's National Committee for Adverse Event Following Immunization. With the estimation that the European Medicines Agency gave, India should have had 320 cases for the 80 million doses already been given. ${ }^{20}$

The North American Food and Drug Administration (FDA) has just suspended injections of the adenoviral Johnson and Johnson/Janssen vaccine after having identified eight cases of CVST with severe thrombocytopenia among close to 7.5 million vaccinated subjects. ${ }^{21-24}$ In their last update of April 21, the FDA reported a total of 12 CVST cases that occurred between 6 to 15 days after Janssen vaccine injection. It would be important to know whether the additional five cases were also associated with thrombocytopenia such as VITT cases. All these cases were in young women with a median age of 37 years (18-59 years old) among 8 million administered doses. ${ }^{18}$

It should be remembered that in the general population, and independent of any vaccination, the annual incidence of venous thrombosis is 1 to 2 per 1,000 people and that of cerebral venous thrombosis is from 1 to 2 per $100,000 .{ }^{4}$ In France, apart from any pandemic, there are around 350 
venous thrombotic episodes per day. The absolute risk of having venous thrombosis after an airplane flight longer than 4 hours was estimated at 1 per 4,600, which therefore appears to be well above (50-100 times) that of having CVST after a SARS-CoV-2 vaccination. ${ }^{25,26}$ Along with the other agencies, it highlights the very significant clinical benefit of vaccination and the very low potential thrombotic risk.

The potential of severe adverse reactions needs also to be weighed against the alternative not to vaccinate. In France, there are currently over 500 people per day admitted to intensive care units with severe or serious forms of COVID-19 and approximately $30 \%$ will die within the following 2 weeks. Despite systematic thromboprophylaxis during hospitalization of patients with COVID-19, the incidence of thrombosis ranges from 7 to $8 \%$ in traditional hospitalization to 25 to $30 \%$ in intensive care. ${ }^{27-29}$ We must also take into account the potential sequelae described in so-called "long COVID" after the hospitalization. ${ }^{30}$

All these data highlight that during infection with SARS-CoV2 and its related disease (COVID-19), thrombosis occurs at least 100 -fold more often without vaccination than after it. Furthermore, politicians, authorities, media, and the public should be reminded that thrombotic risks have been willingly accepted in modern lifestyles. Apart from the already discussed risk from long-distance flights, millions of women use birth control pills which increase the risk of thrombotic events considerably (3-5 times). In fact, vaccination does not appear to induce a higher risk of thrombosis than that reported for combined oral contraceptives. A recent Danish study also notes that the number of cases of thrombosis reported after SARS-CoV-2 vaccination remains below the expected number in the general population, which was estimated from the incidence rate of "classical" venous thrombosis in the entire Danish population before introduction of the vaccination program (91 venous thrombotic episodes per week in individuals 18-64 years old, or 169 episodes per week in individuals $18-99$ years old). ${ }^{31}$ However, we must be careful to compare the thrombotic risks from flying and contraceptive pill intake with the risks of these severe CVST. Clearly the morbi-mortality is different from classical deep venous thrombosis with a high mortality rate reaching around $40 \%$ in VITT. This fatality rate is particularly high because usually the prognosis of classical CVST is less severe with a mortality rate around 5 to $10 \%{ }^{32}$ The Pharmacovigilance Risk Assessment Committee (PRAC) uses O/E ratios as the first level of evaluation of safety signals and these have clearly shown an increase for CVST and SVT in specific age categories.

In countries that have rapidly applied a wide-scale effective vaccination program (Israel, UK, and United States), the COVID-19-related morbidity and mortality have been dramatically reduced, saving at least hundreds of lives per day. This spectacular achievement should be weighed against the extremely low risk of thrombotic events postvaccination.

\section{Hemorrhage and Thrombocytopenia: Rare Events}

Another point to be incorporated in the thrombotic risk analysis is the risk of hemorrhagic incidents. Out of more than 30 million vaccinated people, the MHRA reported 267 hemorrhagic events (including 6 fatal) with the AstraZeneca vaccine and 220 events ( 9 fatal) with the BioNTech/Pfizer vaccine. In the VAERS database in the United States, out of more than 110 million vaccinated people, 439 hemorrhagic episodes were recorded with the BioNTech/Pfizer and Moderna vaccines. ${ }^{18}$ With regard to thrombocytopenia, approximately 60 cases (including 2 fatal) were reported in UK with the AstraZeneca vaccine and 34 cases ( 1 fatal) with the BioNTech/Pfizer vaccine, whereas in the United States only 105 cases of thrombocytopenia were reported with the BioNTech/Pfizer and Moderna vaccines. Immune thrombocytopenic purpura (ITP) is of course possible, as after any vaccination.

The main problem is that there are no trustworthy denominators stratified for age and for sex. ${ }^{8,9}$ Studies are underway to try to better identify the profile of at-risk individuals and to better manage these risks. The collection method for these adverse events is also important since it may be based on spontaneous reporting (with a risk of underreporting) or on more systematic prospective analyses. This continuous and rigorous pharmacovigilance remains crucial for postmarketing (phase 4) vaccination studies. In this health emergency, it should enable us to reinforce the trust established during phase 3 randomized clinical trials and to combat drug mistrust with more extensive experience.

\section{VITT: A Pathophysiological Approach to Thrombosis Following SARS-CoV-2 Vaccine}

The clinical and biological profile that is characteristic of the events described by the German and Norwegian authors and that associates significant thrombocytopenia with major hypercoagulability suggests an immunological mechanism, such as those described during catastrophic antiphospholipid syndrome or heparin-induced thrombocytopenia (HIT). As these patients vaccinated for SARS-CoV-2 had not received heparin, it could be a form of autoimmune HIT or "spontaneous" HIT. ${ }^{33,34}$ A new syndrome was proposed, vaccine-induced prothrombotic immune thrombocytopenia (VIPIT), which has now been changed to VITT. ${ }^{13}$ The German authors, by studying the serum of 9 of the 11 patients, were able to demonstrate very high levels of heparin-platelet-factor 4 (PF4) antibodies in some of them and the ability of these antibodies to activate the platelets of control subjects with or without added PF4. ${ }^{13}$ Furthermore, no addition of heparin was required to activate platelets, which distinguishes VITT of classical HIT. This platelet-activating ability was neutralized in the presence of high heparin concentrations, as in HIT. It was also blocked by the use of monoclonal antibodies binding on the platelet membrane FcrRIIa receptor (CD32a), which induces signaling and platelet aggregation/secretion, platelet-neutrophil and monocyte complex formation, and thrombin generation. Use of high concentrations of polyclonal immunoglobulins can also interrupt the platelet-activating effects of these autoreactive anti-PF4 antibodies, thus confirming immunological hyperactivation of platelets through CD32a. ${ }^{35}$ This profile was also reported in the Norwegian and English cohorts. ${ }^{12,14}$ The 
appearance of these autoantibodies in cases of exaggerated inflammatory response probably by the adenoviral vector, which triggers the release of PF4 contained in the platelets, could be responsible for multicellular activation with massive generation of thrombin, platelet consumption, and severe thrombogenicity. ${ }^{36}$ The inflammatory reaction can cause "NETosis" or "immunothrombosis" with the release of leukocytic DNA, which supports the formation of microthrombi. ${ }^{37-39}$ Disproportionate postvaccination inflammation can also increase endothelial adhesiveness and the release of tissue factor, a real trigger of the thrombin generation, a key enzyme in coagulation. ${ }^{33,36}$ An important point is that this thrombotic and thrombocytopenic symptomatology occurred after the first vaccine injection, with intervals to detection ranging from 4 to 28 days. ${ }^{40}$ The cause and effect relationship has not been clearly established given that postvaccination seroconversion has not been proven and that this type of anti-PF4 antibody can exist prior to the vaccination. Indeed, 5 to $7 \%$ of blood donors have detectable anti-PF4/heparin antibodies. $^{41}$

Of note, particular care should be taken to ensure that these reported cases of thrombosis are not related to a SARSCoV-2 infection concomitant with the vaccination. Not all the reported patients were tested for other immune and systemic conditions that might be responsible for complement pathway activation, inflammation, and coagulation to explain an idiosyncratic reaction. ${ }^{36}$ All UK patients had a negative SARS-CoV-2 polymerase chain reaction at their admission and no recent asymptomatic SARS-CoV-2 infection that may have caused the excessive autoimmune response reported. ${ }^{12}$ The possibility of catastrophic antiphospholipid syndrome cannot also be ruled out, although postinfectious antiphospholipid antibodies are generally less thrombogenic and transient.

\section{Principle of Protection}

As rightfully claimed, "abstention is not a solution!" All scientific societies and thrombosis experts stress the value of continuing vaccination programs to protect patients against serious forms of COVID-19 and to slow viral circulation, particularly of the variants.

Without vaccination, patients are exposed to contracting SARS-CoV-2 with far greater inflammatory and immune stimuli and potentially more devastating consequences than those of the vaccine. ${ }^{42}$ They therefore need to be protected from COVID-19, a disease with particular vascular tropism, through vaccination and subsequent monitoring. ${ }^{43,44}$ In UK, the health authorities and the Joint Committee on Vaccination and Immunisation maintained the second injection programs with the same vaccine for all patients who had received their first AstraZeneca injection with no particular concerns. ${ }^{8}$ In contrast, authorities restrict the use of AstraZeneca now to patients older than 60 years in Germany, 55 years in France, 40 years in Canada, or 30 years in UK, and even recommend using non-AstraZeneca alternatives as booster for younger patients initiated on AstraZeneca. ${ }^{15}$ This recommendation is bare of any evidence (both for efficacy and safety of such an approach) and demonstrates the turmoil caused by the rare thrombotic side effects of the vaccine.

Rapid protection needs to be afforded to patients under 60 years of age with comorbidities (cancer, cardiovascular disease, kidney or liver impairment, immunosuppressant use, obese, diabetes, etc.). This should also be the case for patients on long-term anticoagulant treatment for antiphospholipid syndrome or other reasons. It is very instructive to look at changes in the curves comparing the number of new cases of COVID-19 before and after vaccination implementation in the health care personnel of Paris Public Hospitals (AP-HP) (more than $50 \%$ vaccinated) versus the general population in the greater Paris region (12\% vaccination rate). It shows a very clear drop in infections of AP-HP personnel during the third wave of infection, whereas the curves were completely identical in the autumn of 2020 during the second wave (-Fig. 2). Repeated and appropriate vaccination will likely be the best solution for combatting this pandemic and its variants. More information on potential risk factors and better understanding with a high level of concern on how the vaccine induces these platelet-activating antibodies are needed but larger and quicker population protection is required. ${ }^{45}$

\section{Principle of Education}

In a recent English report on accidents, the annual risk of death from road accidents was estimated at 110 out of $1,000,000$ individuals at the age of 25 years, and 180 out of 1,000,000 at the age of 55 years. ${ }^{46}$ As a comparison, the risk of having a serious event in relation to SARS-CoV-2 vaccination was estimated at 11 out of $1,000,000$ individuals at the age of 25 years, and 4 out of $1,000,000$ at the age of 55 years. ${ }^{46} \mathrm{HIT}$ is a paradoxical prothrombotic syndrome with life-threatening consequences. ${ }^{47}$ Concerning the incidence of HIT in heparinized patients, this varies according to the clinical context and ranges from $0.1 \%$ in medicine with low-molecular-weight heparin to over $3 \%$ in cardiac surgery with unfractionated heparin. ${ }^{47}$ Given its proven clinical benefit, the use of heparin is not prohibited, but patients and their platelet counts are closely monitored to limit these risks.

We must observe, analyze, and make decisions on the basis of our experience and data from real-world prescribing conditions (apart from clinical trials). Medicine is based on scientific evidence and clinical examination. After the reporting and description of thrombosis cases, we were able to have an etiopathogenic lead within several days. The aim is to provide a practical approach to limit the potential risks of vaccination within the general population. The expected benefits of vaccination are far superior to the risks involved (- Table 1A, B). ${ }^{48}$ The benefits of SARS-CoV-2 vaccination remain acknowledged worldwide, especially for avoiding the serious forms, significantly limiting the number of hospitalizations, reducing viral transmission, and providing herd immunity.

There should be more awareness about the approach to those not yet vaccinated, to massmedia and to other opinion leaders (teachers, supervisors at large workplaces, religious leaders) regarding reassurance and promotion of 


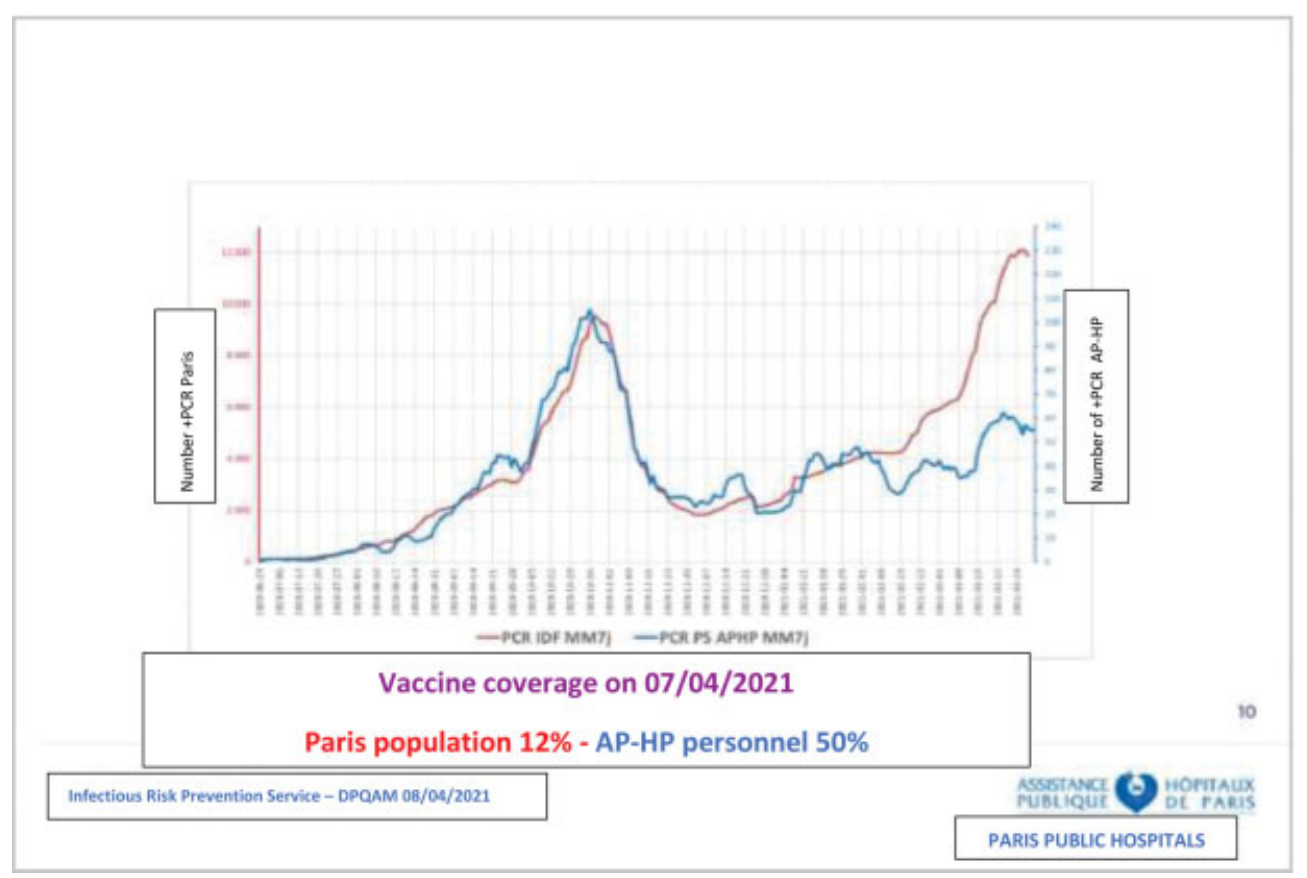

Fig. 2 Profile of changes in COVID-19 cases in the greater Paris region and in AP-HP hospital personnel. Comparing the infection rates between the two populations, the effectiveness of vaccination campaign of AP-HP health workers in hospitals is obvious. After a perfect overlap of both population curves during the previous wave, a significant gap is observed. This is probably related to vaccination campaign acceleration involving more health workers with more than $50 \%$ effectively vaccinated compared with only $12 \%$ in the general Parisian population.

Table 1 Modeling of the risk/benefit ratio of the AstraZeneca vaccine per 100,000 people based on age and infectious risk of exposure $^{48}$

\begin{tabular}{|c|c|c|c|c|}
\hline \multicolumn{5}{|l|}{ A } \\
\hline \multirow[t]{2}{*}{$\begin{array}{l}\text { Potential risk of severe thrombotic } \\
\text { thrombocytopenia, } n / 100,000\end{array}$} & \multirow[t]{2}{*}{ Age (y) } & \multicolumn{3}{|c|}{$\begin{array}{l}\text { Potential benefit (ICU admissions avoided based on } \\
\text { SARS-CoV-2 infection rate) }\end{array}$} \\
\hline & & Low $(55 / 100,000)$ & Medium $(400 / 100,000)$ & High $(900 / 100,000)$ \\
\hline 1.9 & $20-29$ & 0 & 3 & 6 \\
\hline 1.8 & $30-39$ & 0 & 5 & 8 \\
\hline 2.1 & $40-49$ & 1 & 10 & 15 \\
\hline 1.1 & $50-59$ & 1 & 15 & 28 \\
\hline 1.0 & $60-69$ & 3 & 28 & 50 \\
\hline 0.5 & $70-79$ & 6 & 39 & 78 \\
\hline 0.4 & +80 & 13 & 29 & 110 \\
\hline \multicolumn{5}{|l|}{ B } \\
\hline \multirow[t]{2}{*}{$\begin{array}{l}\text { Potential risk of severe thrombotic } \\
\text { thrombocytopenia } n / 100,000\end{array}$} & \multirow[t]{2}{*}{ Age $(y)$} & \multicolumn{3}{|c|}{$\begin{array}{l}\text { Potential benefit (COVID-19 deaths avoided based on } \\
\text { SARS-CoV- } 2 \text { infection rate) }\end{array}$} \\
\hline & & Low $(55 / 100,000)$ & Medium $(400 / 100,000)$ & High $(900 / 100,000)$ \\
\hline 1.9 & $20-29$ & 0 & 0 & 0 \\
\hline 1.8 & $30-39$ & 0 & 2 & 3 \\
\hline 2.1 & $40-49$ & 1 & 7 & 10 \\
\hline 1.1 & $50-59$ & 1 & 8 & 14 \\
\hline 1.0 & $60-69$ & 3 & 25 & 45 \\
\hline 0.5 & $70-79$ & 14 & 87 & 172 \\
\hline 0.4 & +80 & 90 & 197 & 733 \\
\hline
\end{tabular}

Abbreviation: ICU, intensive care unit.

Note: Numbers of cases balancing the risk between potential severe vaccine-related side effects and vaccine-related benefit avoiding ICU admissions (A) and COVID-19-related death (B) in correlation with the age and the importance of SARS-CoV-2 infection exposure. 
vaccination. We should inform primary care and emergency physicians and nurses who may come in contact with people vaccinated and reporting suspicious symptoms on how to identify and appropriately manage VITT. Various guidance is thus proposed by experts and scientific societies for both clinicians and patients. ${ }^{39,49,50}$ This understandable information must be vulgarized and shared as much as possible to make everyone aware but not scared. Therefore widespread, comprehensive, and simplified information should be proposed such as it was done for stroke recognition for example with "BEFAST" acronym:

- B - Balance: watch for a sudden loss of balance with severe headache or dizziness.

- E - Eyes: double vision or blurred vision that does not go away when you blink your eyes.

- F - Fainting: or loss of consciousness.

- A - Abdominal pain: severe and persistent pain, diarrhea, nausea, vomiting, bloody or tarry stools.

- S - Swelling: edema of an arm or a leg with or without color change, shortness of breath with chest pain.

- T - Time: these symptoms appear between 4 and 28 days after vaccination. They can be associated with thrombosis and thrombocytopenia.

Combining this clinical suspicion with a more rapid identification and an adapted treatment implementation would help to re-establish the confidence in the vaccination strategy and to reduce the risk of dying from this rare VITT syndrome.

\section{Principle of Vigilance}

Do all SARS-CoV-2 vaccines carry a risk of thrombosis? Dozens of COVID-19 vaccine candidates of various types are under development, including inactivated, live-attenuated, viral vector, and nucleic acid-based forms. It appears that the methods of vaccination using adenoviruses and containing genetic material from the spike protein are the most likely to result in an inflammatory reaction and systemic stimulation with general symptoms: pain and tenderness at the injection site, headache, tiredness, muscle pain, general feeling of being unwell, chills, fever, joint pain, and nausea ${ }^{8,9}$ (-Table 2).
Vigilance should be maintained regarding large-scale injections of vaccines, which are also based on the use of various adenoviral vectors. No information on any thrombotic risk with the Sputnik V vaccine using different adenoviruses in each injection both of which carrying the gene for SARS-CoV-2 full-length glycoprotein Spike (rAd26-S and rAd5-S) has been published so far. The question is raised as to whether single-strand messenger RNA vaccines, which form antibodies against the spike protein, are more targeted and with fewer vascular side effects. In fact, rare cases of thrombosis have also been reported in patients vaccinated with these mRNAvaccines. There is no "zero risk," and it is essential that we maintain oversight of all patients regardless of the vaccine used. In France, the French General Medical Council (CNOM) obtained important safeguards for protecting physicians in their decision-making to offer the vaccine to their patients. ${ }^{51}$ Article L.3131-15 of the French Public Health Code offers both vaccinated individuals and health care professionals the same legal safeguards as those provided for in the context of compulsory vaccinations. ${ }^{52}$ Full compensation for any accidents attributable to the vaccination campaign will therefore be assured by the Office National d'Indemnisation des Accidents Médicaux (French National Office of Medical Workers Compensation) in the name of national solidarity.

\section{Principle of Precaution}

We need to inform, reassure, and support our patients when the prescription is made. Based on these observations, we propose a tracking algorithm for vaccinated patients. It uses a 10 -point guideline for safe decision-making.

1. Intramuscular injection should be done correctly in the deltoid muscle and not intravascularly by using the right technique and applying the injection at the appropriate lower site of the muscle. ${ }^{53,54}$ Injection itself can cause injuries as it is not as harmless as one commonly thinks.

2. Check that there is not an extensive ecchymotic or purpuric local reaction that is particularly painful.

3. Be aware of the possibility of minimal systemic signs, low-grade fever, or muscular pain, which relate to the

Table 2 The different types of vaccines used in Europe: classical side effects ${ }^{9,10}$

\begin{tabular}{|l|l|l|l|}
\hline Type of Vaccine & $\mathbf{1 / 1 0}$ vaccinated patients & $\mathbf{1 / 1 0 0}$ vaccinated patients & Allergic reactions \\
\hline $\begin{array}{l}\text { BioNTech/Pfizer } \\
\text { (mRNA) }\end{array}$ & $\begin{array}{l}\text { Local pain, swelling, fatigue, headache, } \\
\text { muscular pain, joint pain, fever }\end{array}$ & $\begin{array}{l}\text { Pain to extremities, } \\
\text { local adenopathy, } \\
\text { poor general wellbeing }\end{array}$ & $\begin{array}{l}11 / 1,000,000 \text { vaccinated } \\
\text { patients: rare cases of } \\
\text { anaphylactic reaction }\end{array}$ \\
\hline $\begin{array}{l}\text { Moderna } \\
\text { Therapeutics } \\
\text { (mRNA) }\end{array}$ & $\begin{array}{l}\text { Local pain, edema, local adenopathy, } \\
\text { fatigue, headache, muscular pain, } \\
\text { nausea, fever }\end{array}$ & $\begin{array}{l}\text { Redness at injection site, } \\
\text { vesicular lesions }\end{array}$ & $\begin{array}{l}2.5 / 1,000,000 \text { vaccinated } \\
\text { patients: rare anaphylactic } \\
\text { reaction }\end{array}$ \\
\hline $\begin{array}{l}\text { Oxford/AstraZeneca } \\
\text { ChAdOx1-S }\end{array}$ & $\begin{array}{l}\text { Local pain, swelling, fatigue, } \\
\text { muscular pain, joint pain, } \\
\text { poor general wellbeing, nausea, fever }\end{array}$ & $\begin{array}{l}\text { Dizziness, sweating, } \\
\text { abdominal pain, skin rash }\end{array}$ & $\begin{array}{l}10 / 1,000,000 \text { vaccinated } \\
\text { patients: rare anaphylactic } \\
\text { reaction }\end{array}$ \\
\hline $\begin{array}{l}\text { Janssen } \\
\text { Ad26.COV2-S }\end{array}$ & $\begin{array}{l}\text { Local pain, headache, fatigue, } \\
\text { muscular pain, joint pain, } \\
\text { poor general wellbeing, nausea, fever }\end{array}$ & $\begin{array}{l}\text { Cough, joint pain, fever, } \\
\text { erythema, edema, chills }\end{array}$ & $\begin{array}{l}\text { Rare anaphylactic reaction, } \\
\text { hypersensitivity, hives }\end{array}$ \\
\hline
\end{tabular}


expected inflammatory response and to stimulation of the immune system, and which varies from one subject to another. It is advisable for the patient to drink a lot of fluids and take paracetamol in case of flu-like symptoms and to discuss it with his doctor. These signs should decrease in 48 to 72 hours.

4. Patients should consult with their doctors urgently or go to the hospital in the event of emerging and persistent clinical manifestations more than 4 days after the vaccination, including intense and persistent headaches, dizziness, visual disorders, impaired speech, acute pain or worsening muscular pain, edema of a limb suggestive of phlebitis, significant changes in the temperature of a limb (heat or cold), difficulty breathing, and sudden heart rate acceleration. The same awareness should be given to unusual bleeding signs, especially petechiae. For effective work-up, patients presenting with suggestive symptoms should be admitted with a low threshold to allow for an immediate and thorough work-up.

5. Start laboratory investigations after physical examination: complete blood count with platelet count, D-dimers $(>1,000 \mathrm{ng} / \mathrm{mL})$, and schistocytes to rule out a hypercoagulable state with platelet consumption (platelets $<120 \mathrm{G} / \mathrm{L}$ ) or disseminated intravascular coagulation with a decrease in fibrinogen $(<2 \mathrm{~g} / \mathrm{L})$ (depending on the clinical profile, additional tests may be ordered such as C-reactive protein, antiphospholipid antibodies [anticardiolipin, antibetaGP1], screening for lupus anticoagulant, antinuclear antibodies, ADAMTS13, etc.).

6. Detect thrombosis through imaging in various sites (venous ultrasound, magnetic resonance imaging, computed tomography angiography).

7. Investigation for HIT in case of thrombocytopenia (platelets $<120 \mathrm{G} / \mathrm{L}$ ) through screening for heparin-PF4 antibodies with ELISA assay (Lifecodes PF4 IgG [Immucor] or Asserachrom HPIA - IgG [Stago]). Assess the ability of these antibodies to activate platelets through a rapid functional test in the presence of PF4 (heparin-induced multi-electrode aggregometry method or adapted flow cytometry specialized test by expert center). ${ }^{55-57}$

8. Implement without delay an effective nonheparin antithrombotic treatment by injectable anticoagulant (fondaparinux, danaparoid, argatroban) based on availability, experience, and the possibilities of close biological monitoring of the treatment. Depending on the clinical context and evolution, the switch to direct oral anticoagulant (dabigatran, rivaroxaban, apixaban) can be proposed.

9. In the event of major thrombotic events, infuse immunoglobulins $(1 \mathrm{~g} / \mathrm{kg})$ in combination with antithrombotics for 48 hours (to occupy the CD32 membrane sites of autoantibody cell docking and thus limit multicellular excitability leading to this generalized prothrombotic event). Steroids and plasma exchange are also options to reduce these incendiary autoantibodies. Interestingly, inhibitors of Bruton's tyrosine kinase, pleiotropically targeting multiple pathways downstream of CD32 activation and approved for B cell malignancies (e.g., ibrutinib), are proposed as another potential therapeutic option in VITT. ${ }^{36}$

10. Report the proven and documented serious event to pharmacovigilance authorities.

After seeing what still needs to be accomplished, let's have a look at what not to do:

1. Systematic management of vaccination with thromboprophylaxis (low-molecular-weight heparin or direct oral anticoagulant or aspirin).

2. Systematic screening for thrombophilia before vaccination.

3. Systematic measure of anti-PF4 antibodies after vaccination.

4. Systematic monitoring of changes in D-dimers before and after vaccination.

5. Systematic use of a venous ultrasound exam before and after vaccination.

6. Contraindicating SARS-CoV-2 vaccination in case of history of thrombosis.

7. Contraindicating SARS-CoV-2 vaccination in case of autoimmune disease.

8. Contraindicating SARS-CoV-2 vaccination in case of history of HIT but due to potential "genetic susceptibility" choosing mRNA vaccine is preferable.

9. Systematically contraindicating SARS-CoV-2 vaccination in case of history of allergy. Of course this is not the case of allergy after first dose of any vaccine.

10. Contraindicating SARS-CoV-2 vaccination in case of immune thrombocytopenia (ITP).

\section{Conclusion}

The scientific evaluation of the European Medicines Agency concluded to the safe and effective use of COVID-19 vaccines and that the most recent data do not change the recommendations from the Pharmacovigilance Risk Assessment Committee (PRAC). Use of the vaccine during national vaccination campaigns must take into account the pandemic situation and the availability of the vaccine in each member state. All vaccines must be administered under close supervision with appropriate medical treatment available. ${ }^{15}$ Most international medical scientific societies, including the International Society on Thrombosis and Haemostasis (ISTH) and the World health Organization (WHO), have issued statements to encourage populations of countries where the AstraZeneca vaccine was available to continue using it. ${ }^{40,49,50,58}$ The vaccination has clear and accessible health, economic, and societal objectives. The role of any preventive medicine is to offer safe protection and control of potential adverse events, although the latter is part of the risk of medical decision-making. Abstinence is not an option since it results in failure to provide assistance to a large population that remains in danger. Action with increased vigilance and a broader understanding is the best solution in our public health mission.

Conflict of Interest

None declared. 


\section{References}

1 WHO. Strategic Advisory Group of Experts (SAGE) on immunization. Accessed May 10, 2021 at: https://www.who.int/immunization/sage/fr/

2 WHO. Ten threats to global health in 2019. https://www.who.int/ news-room/spotlight/ten-threats-to-global-health-in-2019. Accessed May 10, 2021

3 Johns Hopkins Coronavirus Disease Resource Center. Accessed April 2021 at: https://coronavirus.jhu.edu

4 World Bank. Global Economic Prospects. Washington, DC: World Bank; 2020

5 Hodgson SH, Mansatta K, Mallett G, Harris V, Emary KRW, Pollard AJ. What defines an efficacious COVID-19 vaccine? A review of the challenges assessing the clinical efficacy of vaccines against SARSCoV-2. Lancet Infect Dis 2021;21(02):e26-e35

6 Poland GA, Ovsyannikova IG, Kennedy RB. SARS-CoV-2 immunity: review and applications to phase 3 vaccine candidates. Lancet 2020;396(10262):1595-1606

7 Cox RJ, Brokstad KA. Not just antibodies: B cells and T cells mediate immunity to COVID-19. Nat Rev Immunol 2020;20 (10):581-582

8 MHRA. Accessed May 10, 2021 at: https://yellowcard.mhra.gov.uk/ 9 Signal assessment report on embolic and thrombotic events (SMQ) with COVID-19 Vaccine (ChAdOx1-S [recombinant]) COVID-19 Vaccine AstraZeneca (Other viral vaccines). Accessed May 25, 2021 at: https://www.ema.europa.eu/en/documents/ prac-recommendation/signal-assessment-report-embolic-thrombotic-events-smq-covid-19-vaccine-chadox1-s-recombinant-covid_en.pdf

10 Meo SA, Bukhari IA, Akram J, Meo AS, Klonoff DC. COVID-19 vaccines: comparison of biological, pharmacological characteristics and adverse effects of Pfizer/BioNTech and Moderna vaccines. Eur Rev Med Pharmacol Sci 2021;25(03):1663-1669

11 Wise J. Covid-19: European countries suspend use of OxfordAstraZeneca vaccine after reports of blood clots. BMJ 2021;372 (699):n699

12 Scully M, Singh D, Lown R, et al. Pathologic antibodies to platelet factor 4 after ChAdOx1 nCoV-19 vaccination. N Engl J Med 2021;384(23):2202-2211

13 Greinacher A, Thiele T, Warkentin TE, Weisser K, Kyrle PA, Eichinger S. Thrombotic thrombocytopenia after ChAdOx1 nCov-19 vaccination. N Engl J Med 2021;384(22):2092-2101

14 Schultz NH, Sørvoll IH, Michelsen AE, et al. Thrombosis and thrombocytopenia after ChAdOx1 $\mathrm{nCoV}-19$ vaccination. $\mathrm{N}$ Engl J Med 2021;384(22):2124-2213

15 EMA. AstraZeneca's COVID-19 vaccine: EMA finds possible link to very rare cases of unusual blood clots with low blood platelets. Accessed May 10, 2021 at: https://www.ema.europa.eu/en/news/ astrazenecas-covid-19-vaccine-ema-finds-possible-link-very-rarecases-unusual-blood-clots-low-blood

16 Mahase E. Covid-19: AstraZeneca vaccine is not linked to increased risk of blood clots, finds European Medicine Agency. BM] 2021;372(774):n774

17 Merchant $\mathrm{H}$. Can post immunisation increase in acute phase proteins explain the recent thrombotic events with CoViD vaccines? BMJ 2021;373:n883

18 NCIRD. Reports of cerebral venous sinus thrombosis with thrombocytopenia after Janssen COVID-19 vaccine. Accessed May 10, 2021 at: https://www.cdc.gov/vaccines/acip/meetings/downloads/ slides-2021-04/03-COVID-Shimabukuro-508.pdf

19 ANSM. Accessed May 10, 2021 at: https://ansm.sante.fr/actualites/retour-dinformation-sur-le-prac-davril-2021

20 India will review claims of AstraZeneca vaccine causing blood clots in 'unusual' sites. Accessed May 25, 2021 at: https://www. firstpost.com/health/india-will-review-claims-of-astrazeneca-vaccine-causing-blood-clots-in-unusual-sites-9527001.html
21 CDC. Accessed May 10, 2021 at:https://www.cdc.gov/media/ releases/2021/s0413-JJ-vaccine.html

22 Muir KL, Kallam A, Koepsell SA, Gundabolu K. Thrombotic thrombocytopenia after Ad26.COV2.S vaccination. N Engl J Med 2021;384(20):1964-1965

23 Sadoff J, Davis K, Douoguih M. Thrombotic thrombocytopenia after Ad26.COV2.S vaccination - response from the manufacturer. N Engl J Med 2021;384(20):1965-1966

24 Ferro JM, Aguiar de Sousa D. Cerebral venous thrombosis: an update. Curr Neurol Neurosci Rep 2019;19(10):74

25 Kuipers S, Cannegieter SC, Middeldorp S, Robyn L, Büller HR, Rosendaal FR. The absolute risk of venous thrombosis after air travel: a cohort study of 8,755 employees of international organisations. PLoS Med 2007;4(09):e290

26 WHO. WHO Research Into Global Hazards of Travel (WRIGHT) Project: final report of phase I. Accessed May 10, 2021 at: https://www.who. int/cardiovascular_diseases/wright_project/phase1_report/WRIGHT \%20REPORT.pdf

27 Jiménez D, García-Sanchez A, Rali P, et al. Incidence of VTE and bleeding among hospitalized patients with coronavirus disease 2019: a systematic review and meta-analysis. Chest 2021;159 (03):1182-1196

28 Nopp S, Moik F, Jilma B, Pabinger I, Ay C. Risk of venous thromboembolism in patients with COVID-19: a systematic review and meta-analysis. Res Pract Thromb Haemost 2020;4(07):1178-1191

29 Roubinian NH, Dusendang JR, Mark DG, et al. Incidence of 30-day venous thromboembolism in adults tested for SARS-CoV-2 infection in an integrated health care system in Northern California. JAMA Intern Med 2021;181(7):997-1000

30 NCE. COVID-19 rapid guideline: managing the long-term effects of COVID-19 - NICE guideline [NG188]. Accessed May 10, 2021 at: https://www.nice.org.uk/GUIDANCE/ng188

31 Østergaard SD, Schmidt M, Horváth-Puhó E, Thomsen RW, Sørensen HT. Thromboembolism and the Oxford-AstraZeneca COVID-19 vaccine: side-effect or coincidence? Lancet 2021;397 (10283):1441-1443

32 Silvis SM, de Sousa DA, Ferro JM, Coutinho JM. Cerebral venous thrombosis. Nat Rev Neurol 2017;13(09):555-565

33 Warkentin, et al. Spontaneous prothrombotic disorder ressembling to HIT. Am J Med 2008;121:632-636

34 Greinacher A, Selleng K, Warkentin TE. Autoimmune heparin-induced thrombocytopenia. J Thromb Haemost 2017;15(11):2099-2114

35 Padmanabhan A, Jones CG, Pechauer SM, et al. IVIg for treatment of severe refractory heparin-induced thrombocytopenia. Chest 2017; 152(03):478-485

36 von Hundelshausen P, Lorenz R, Siess W, Weber C. Vaccineinduced immune thrombotic thrombocytopenia (VITT): targeting pathomechanisms with Bruton tyrosine kinase inhibitors. Thromb Haemost 2021. Doi: 10.1055/a-1481-3039

37 McGonagle D, O'Donnell JS, Sharif K, Emery P, Bridgewood C. Immune mechanisms of pulmonary intravascular coagulopathy in COVID-19 pneumonia. Lancet Rheumatol 2020;2(07):e437-e445

38 Merad M, Martin JC. Pathological inflammation in patients with COVID-19: a key role for monocytes and macrophages. Nat Rev Immunol 2020;20(06):355-362

39 Yu J, Yuan X, Chen H, Chaturvedi S, Braunstein EM, Brodsky RA. Direct activation of the alternative complement pathway by SARS-CoV-2 spike proteins is blocked by factor D inhibition. Blood 2020;136(18):2080-2089

40 ISTH. ISTH statement on AstraZeneca COVID-19 vaccine and thrombosis. Accessed May 10, 2021 at: https://www.isth.org/ news/556057/ISTH-Statement-on-AstraZeneca-COVID-19-Vaccine-and-Thrombosis.htm

41 Hursting MJ, Pai PJ, McCracken JE, et al. Platelet factor 4/heparin antibodies in blood bank donors. Am J Clin Pathol 2010;134(05): 774-780 
42 Gencer S, Lacy M, Atzler D, van der Vorst EPC, Döring Y, Weber C. Immunoinflammatory, thrombohaemostatic, and cardiovascular mechanisms in COVID-19. Thromb Haemost 2020;120(12): 1629-1641

43 Lippi G, Sanchis-Gomar F, Favaloro EJ, Lavie CJ, Henry BM. Coronavirus disease 2019-associated coagulopathy. Mayo Clin Proc 2021;96(01):203-217

44 Siddiqi HK, Libby P, Ridker PM. COVID-19 - a vascular disease. Trends Cardiovasc Med 2021;31(01):1-5

45 Cines DB, Bussel JB. SARS-CoV-2 vaccine-induced immune thrombotic thrombocytopenia. N Engl J Med 2021;384(23):2254-2256

46 Cuffe R. AstraZeneca vaccine: How do you weigh up the risks and benefits? Accessed May 10, 2021 at: https://www.bbc.com/news/ explainers-56665396

47 Sahu KK, Jindal V, Anderson J, Siddiqui AD, Jaiyesimi IA. Current perspectives on diagnostic assays and Anti-PF4 antibodies for the diagnosis of heparin-induced thrombocytopenia. J Blood Med 2020;11:267-277

48 EMA. European Medicines Agency Annex to Vaxzevria Art.5.3 - Visual risk contextualisation. Accessed May 10, 2021 at: https://www.ema. europa.eu/en/documents/chmp-annex/annex-vaxzevria-art53-visual-risk-contextualisation_en.pdf

49 Thrombosis Canada. Vaccine-induced prothrombotic immune thrombocytopenia (VIPIT). Accessed May 10, 2021 at: https:// thrombosiscanada.ca/wp-uploads/uploads/2021/04/51.-Vaccineinduced-prothrobotic-immune-thrombcytopenia_02April2021.pdf

50 Guidance produced by the Expert Haematology Panel (EHP) focussed on Vaccine induced Thrombosis and Thrombocytopenia (VITT). Accessed May 25, 2021 at: https://b-s-h.org.uk/about-us/ news/guidance-produced-by-the-expert-haematology-panel-ehpfocussed-on-vaccine-induced-thrombosis-and-thrombocytopenia-vitt/
51 https://www.conseil-national.medecin.fr/sites/default/files/external-package/bulletin/1v5iz4m/medecins_71.pdf

52 Responsibility of physicians in the vaccine decision. Accessed May 25, 2021 at: https://www.conseil-national.medecin.fr/publications/communiques-presse/responsabilite-medecins-decisionvaccinale

52 Accessed May 10, 2021 at:https://www.conseilnational. medecin.fr/publications/responsabilite-medecins-decisionvaccinale

53 Nakajima Y, Mukai K, Takaoka K, et al. Establishing a new appropriate intramuscular injection site in the deltoid muscle. Hum Vaccin Immunother 2017;13(09):2123-2129

54 Behrens RH, Patel V. Avoiding shoulder injury from intramuscular vaccines. Lancet 2021;397(10273):471

55 contrib-group>Galea VV Galea, Khaterchi AA Khaterchi, Robert FF Robert, Gerotziafas GG Gerotziafas, Hatmi MM Hatmi, Elalamy II Elalamy. Heparin-induced multiple electrode aggregometry is a promising and useful functional tool for heparin-induced thrombocytopenia diagnosis: confirmation in a prospective study. Platelets 2013;24(06):441-447

56 Morel-Kopp MC, Mullier F, Gkalea V, et al; subcommittee on platelet immunology. Heparin-induced multi-electrode aggregometry method for heparin-induced thrombocytopenia testing: communication from the SSC of the ISTH. J Thromb Haemost 2016;14(12):2548-2552

57 Tardy-Poncet B, Montmartin A, Piot M, et al; On Behalf of The Gfht-Hit Study Group. Functional flow cytometric assay for reliable and convenient heparin-induced thrombocytopenia diagnosis in daily practice. Biomedicines 2021;9(04):332

58 WHO. COVID-19 vaccines. Accessed May 10, 2021 at: https:// www.who.int/emergencies/diseases/novel-coronavirus-2019/ covid-19-vaccines 